\title{
Development of a hematopoietic prostaglandin D synthase-degradation inducer
}

Hidetomo Yokoo, ${ }^{a, b, \#}$ Norihito Shibata, ${ }^{c, d, \#}$ Miyako Naganuma, ${ }^{a}$ Kiyonaga Fujii, ${ }^{e}$ Takahito Ito, ${ }^{a}$ Kosuke Aritake, ${ }^{f, *}$ Mikihiko Naito, ${ }^{a, *}$ and Yosuke Demizu ${ }^{a, b, *}$

${ }^{a}$ Division of Organic Chemistry, National Institute of Health Sciences, 3-25-26, Tonomachi, Kawasaki, Kanagawa, 210-9501, Japan; ${ }^{b}$ Graduate School of Medical Health Sciences, Yokohama City University, Yokohama, Kanagawa, 230-0045, Japan; ${ }^{c}$ Division of Molecular Target and Gene Therapy Products, National Institute of Health Sciences, 3-25-26, Tonomachi, Kawasaki, Kanagawa, 210-9501, Japan; ${ }^{d}$ Division of Biochemistry, National Institute of Health Sciences, 3-25-26, Tonomachi, Kawasaki, Kanagawa, 210-9501, Japan; ${ }^{\prime}$ Laboratory of Analytical Chemistry, Daiichi University of Pharmacy, 22-1 Tamagawa-cho, Minami-ku, Fukuoka, 815-8511, Japan; ${ }^{\text {LLaboratory }}$ of Chemical Pharmacology, Daiichi University of Pharmacy, 22-1 Tamagawa-cho, Minami-ku, Fukuoka, 815-8511, Japan.

Keywords: prostaglandin D2; ubiquitin-proteasome system; protein knockdown; PROTACs

Corresponding author:

Tel: +81-44-270-6578, Fax: +81-44-270-6578, E-mail: demizu@nihs.go.jp (Y. Demizu) Tel: +81-44-270-6600 (Ex. 3210), E-mail: miki-naito@nihs.go.jp (M. Naito)

Tel: +81-92-541-0168 ( Ex. 416 ) , Fax: +81-92-553-5698, E-mail: k-aritake@daiichi-cps.ac.jp (K. Aritake) 
\#These authors contributed equally to this study. 


\begin{abstract}
Although hematopoietic prostaglandin D synthase (H-PGDS) is an attractive target for treatment of a variety of diseases, including allergic diseases and Duchenne muscular dystrophy, no H-PGDS inhibitors have yet been approved for treatment of these diseases. Therefore, the development of novel agents having other mode of actions to modulate the activity of H-PGDS is required. In this study, a chimeric small molecule that degrades H-PGDS via the ubiquitin-proteasome system, PROTAC(H-PGDS)-1, was developed. PROTAC(H-PGDS)-1 is composed of two ligands, TFC-007 (that binds to H-PGDS) and pomalidomide (that binds to cereblon). PROTAC(H-PGDS)-1 showed potent activity in the degradation of H-PGDS protein via the ubiquitin-proteasome system and in the suppression of prostaglandin $\mathrm{D}_{2}\left(\mathrm{PGD}_{2}\right)$ production. Notably, PROTAC(H-PGDS)-1 was slightly more effective in the suppression of $\mathrm{PGD}_{2}$ production than the known inhibitor, TFC-007. Thus, the H-PGDS degrader-PROTAC(H-PGDS)-1-is expected to be useful in biological research and clinical therapies. (145 words)
\end{abstract}


Overproduction of $\mathrm{PGD}_{2}$ is related to a variety of diseases, including allergic diseases, ${ }^{1,2}$ physiological sleep, ${ }^{3}$ and Duchenne muscular dystrophy. ${ }^{4}$ H-PGDS is one of the enzymes involved in $\mathrm{PGD}_{2}$ synthesis; therefore, H-PGDS is a potential therapeutic target for such diseases, for example, in the nasal mucosa of patients with allergic rhinitis. ${ }^{5}$ In vivo studies have demonstrated that H-PGDS inhibition is effective in the treatment of allergic inflammation. ${ }^{6-8}$ To date, several types of H-PGDS inhibitors have been developed as therapies for allergic and inflammatory responses. However, the advancement of these inhibitors into clinical studies has not been satisfactory. Thus, the development of novel agents for clinical investigation with modes of action other than H-PGDS inhibition is required.

In recent years, innovative chimeric drugs, PROTACs (proteolysis targeting chimeras) and SNIPERs (specific and non-genetic inhibitor of apoptosis protein [IAP]-dependent protein erasers), which enable the degradation of target proteins via the ubiquitin-proteasome system (UPS), have been developed. These drugs are chimeric molecules, composed of target protein ligands and E3 ligase ligands, which recruit a target protein in proximity to an E3 ligase to induce protein degradation. ${ }^{9-11}$ Some degraders have been reported to show more durable suppression of the cellular responses caused by the target protein than small molecule inhibitors. ${ }^{12-14}$ A variety of PROTACs and SNIPERs have been developed for the treatment of cancer that targeted related proteins, such as transcriptional regulators, nuclear receptors, and protein kinases. ${ }^{11,15,16}$ As a new mechanism of action for regulating the activity of H-PGDS, the development of degraders targeting the H-PGDS protein is an attractive approach for the treatment of chronic allergic diseases. In the present study, we developed the chimeric small molecule PROTAC(H-PGDS)-1, which had potent activity for the 
degradation of H-PGDS protein via the UPS and in the suppression of $\mathrm{PGD}_{2}$ production.

\section{Results and Discussion}

Among the representative H-PGDS selective inhibitors, HQL-79, ${ }^{16}$ F092, ${ }^{18}$ BSPT, ${ }^{19}$ TAS-204, ${ }^{7}$ and TFC-007, TAS-204, and TFC-007 show high activity (with $\mathrm{IC}_{50}$ values of 23 and $83 \mathrm{nM}$, respectively, Figure 1a). ${ }^{5-8}$ The binding mode between H-PGDS and F092 (and HQL-79) has been revealed by X-ray diffraction. ${ }^{17,18}$ From the above information, we focused on the chemical structures of F092 and TFC-007, which contain the same $N$-phenyl-5-pyrimidinecarboxamide moiety, to design a chimeric molecule. In the X-ray crystal structure of the ligand binding domain of H-PGDS and F092 (PDB: 5YWX), the $N$-phenyl-5-pyrimidinecarboxamide moiety faces to the inside and the 2-pyrrolidone moiety is orientated to the outside of the protein (Figure 1b). We hypothesized that the elongated structure of TFC-007, similar to F092, would be suitable as a target protein ligand and the morpholine moiety of TFC-007 would face the surface of the protein. Thus, we designed a TFC-007-based PROTAC by the replacement of the morpholine moiety with a piperazine moiety for linking with the E3 ligase ligand. As the E3 ligase ligand, pomalidomide was selected, which binds to cereblon (CRBN). CRBN is a broadly expressed protein and forms part of the cullin-4-containing E3 ubiquitin ligase complex (CRL4). The structures of PROTAC(H-PGDS)-1 and the negative control PROTAC(H-PGDS)-2 with $N$-methylated pomalidomide, which is considered to have considerably reduced binding affinity to $\mathrm{CRBN},{ }^{12}$ are shown in Figure 1c. The synthetic routes for 
PROTAC(H-PGDS)-1 and PROTAC(H-PGDS)-2 are given in the supporting information (Schemes S1 and S2, respectively).

(a)

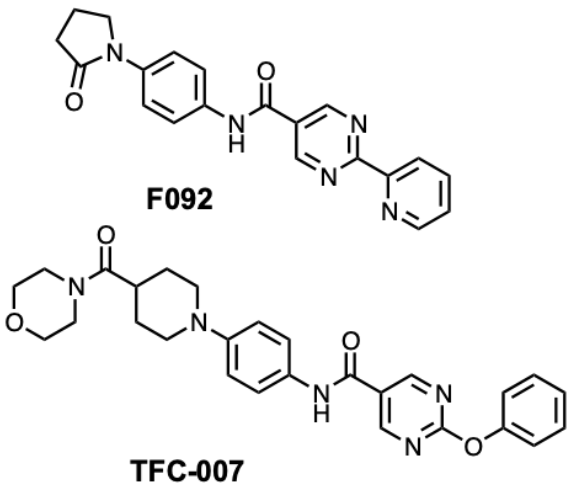

(b)

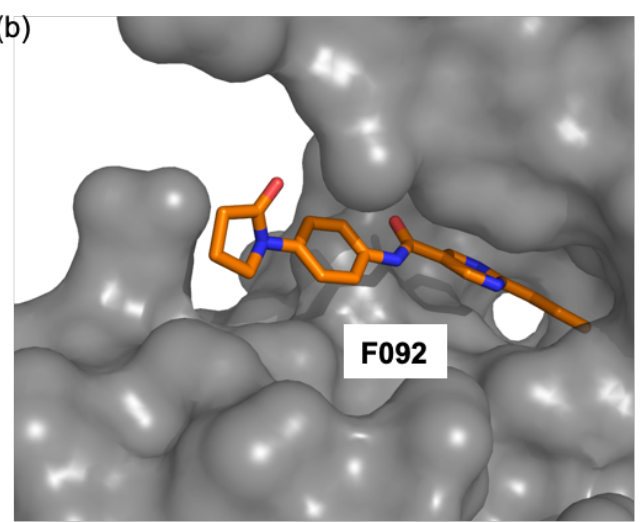

(c)<smiles>[R]N1C(=O)CCC(N2C(=O)c3cccc(NCCOCCOCCOCCOCCOCCC(=O)N4CCN(C(=O)C5CCN(c6ccc(NC(=O)c7cnc(Oc8ccccc8)nc7)cc6)CC5)CC4)c3C2=O)C1[R]([H])=C</smiles>

Figure 1. (a) Chemical structures of the H-PGDS inhibitors F092 and TFC-007. (b) X-ray crystal structure of H-PGDS with F092 (PDB: 5YWX). (c) Chemical structures of PROTAC(H-PGDS)-1 and PROTAC(H-PGDS)-2.

To examine the effect of PROTAC(H-PGDS)-1 on H-PGDS protein levels, human KU812 cells expressing H-PGDS protein were treated with graded concentrations of PROTAC(H-PGDS)-1 for $3 \mathrm{~h}$ (Figure 2a). Effective reduction of H-PGDS protein by PROTAC(H-PGDS)-1 was observed at concentrations $\geq 10 \mathrm{nM}$ and the maximum activity was observed at 100-1000 nM. Additionally, 
PROTAC(H-PGDS)-1, at concentrations $\geq 10 \mathrm{nM}$, showed more potent activity in the reduction of the H-PGDS protein when the cells were incubated with PROTAC(H-PGDS)-1 for 6 or 24 h (Figure 2a). Similar protein reduction activity for PROTAC(H-PGDS)-1 was also observed in MEG-01s cells expressing H-PGDS protein (Figure S1). This reduction activity was not observed for SNIPER(H-PGDS)-1, in which TFC-007 is conjugated to an IAP ligand LCL161 derivative (Figure S2). While PROTAC(H-PGDS)-1 showed potent activity in H-PGDS protein reduction, PROTAC(H-PGDS)-1 had little effect on the protein levels of mPGES-1, one of the major enzymes for $\mathrm{PGE}_{2}$ synthesis (Figure S3), ${ }^{20}$ suggesting that PROTAC(H-PGDS)-1 may specifically reduce H-PGDS and not other prostaglandin (PG) synthases.

To understand the mechanism for the reduction of H-PGDS protein by PROTAC(H-PGDS)-1, we examined the turnover of H-PGDS protein after PROTAC(H-PGDS)-1 treatment. When KU812 cells were treated with the protein synthesis inhibitor cycloheximide in KU812 cells, the levels of H-PGDS protein were dramatically decreased within $6 \mathrm{~h}$ in the PROTAC(H-PGDS)-1-treated cells, but were retained in the control cells (Figure 2b). In contrast, the turnover rate of cyclin B1 was not affected by PROTAC(H-PGDS)-1 treatment. These results indicated that PROTAC(H-PGDS)-1 selectively induces a reduction in H-PGDS protein levels. Furthermore, the levels of $H-P G D S$ mRNA in KU812 cells were not affected by PROTAC(H-PGDS)-1 (Figure 2c). These results indicated that PROTAC(H-PGDS)-1 induces the degradation of H-PGDS protein. 
(a)

$3 \mathbf{h}$

PROTAC(H-PGDS)-1
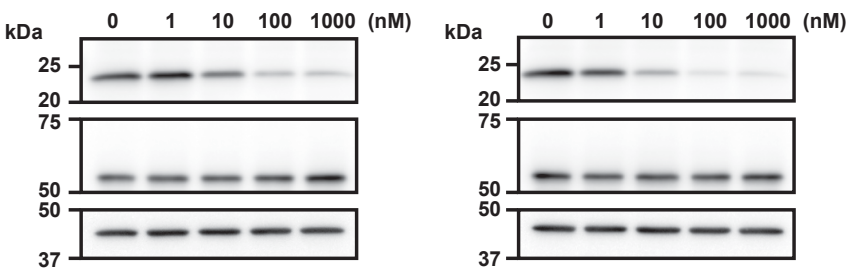

PROTAC(H-PGDS)-1
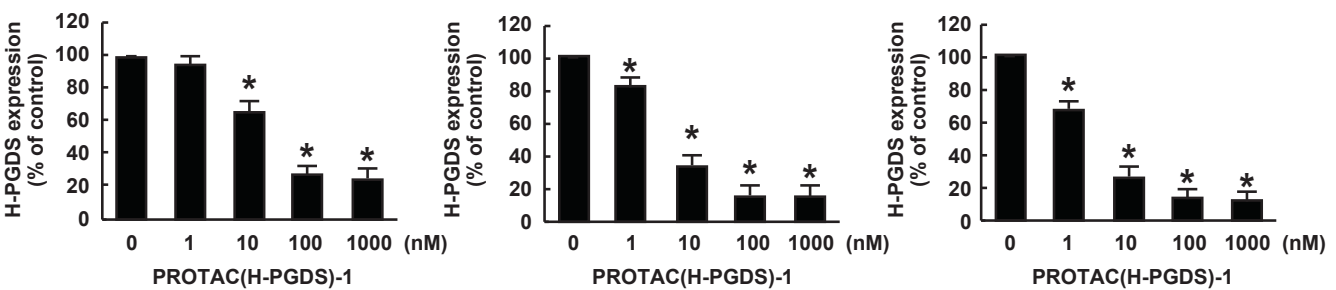

(b)
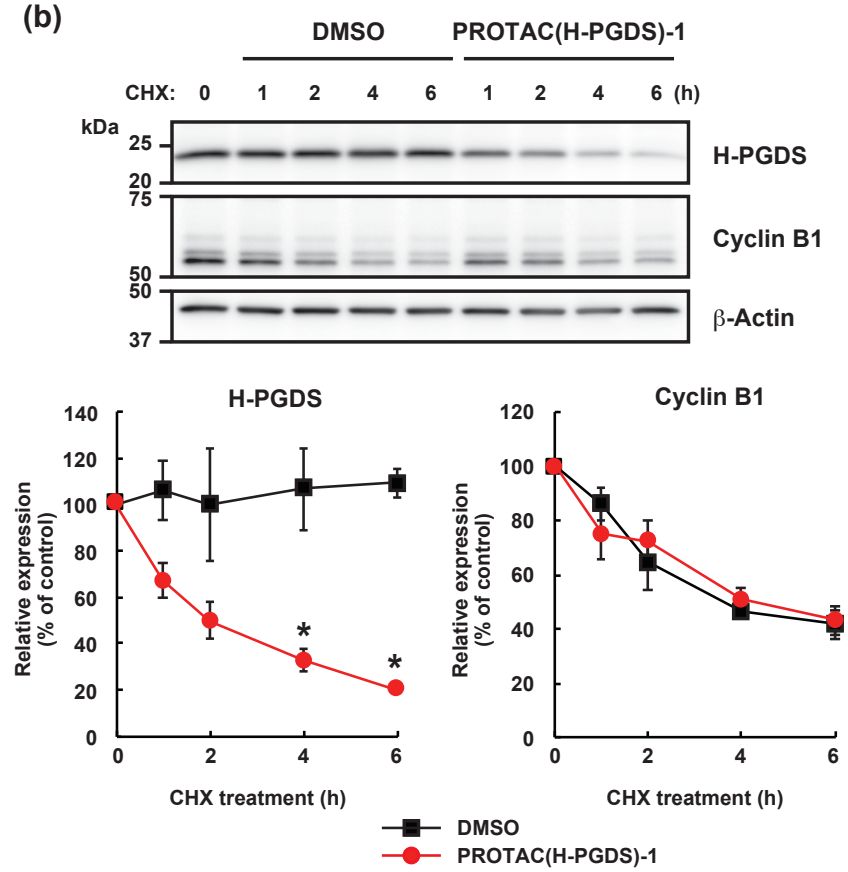

(c)

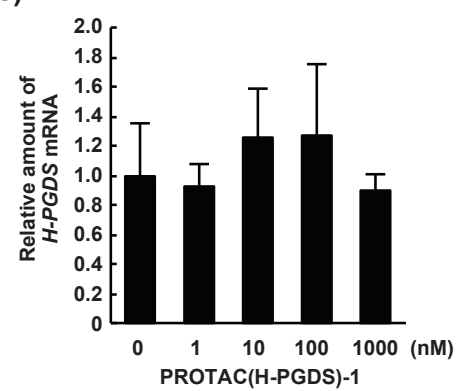

Figure 2. PROTAC(H-PGDS)-1 is a degrader for H-PGDS protein. (a) KU812 cells were incubated with the indicated concentration of PROTAC(H-PGDS)-1 for the indicated time. The H-PGDS/ $\beta$-Actin ratio was normalized by the vehicle control as 100 . Data in the bar graph are the mean $\pm \mathrm{SD}(n=3)$. (b) Turnover of H-PGDS protein in KU812 cells after treatment with $10 \mu \mathrm{g} / \mathrm{ml}$ of cycloheximide (CHX) in the presence or 
absence of $100 \mathrm{nM}$ of PROTAC(H-PGDS)-1 for the indicated periods. The H-PGDS/ $\beta$-Actin and cyclin B1/ $\beta$-Actin ratios were normalized by the time 0 control as 100. Data in the graphs are the mean $\pm \mathrm{SD}(n=3)$. (c) Expression of $H-P G D S$ mRNA in KU812 cells. Cells were incubated with the indicated concentration of PROTAC(H-PGDS)-1 for $6 \mathrm{~h}$. Expression levels are relative to vehicle treatment, which was arbitrarily set to 1 . Data in the bar graph are the mean $\pm \mathrm{SD}(n=3)$. ${ }^{*} P<$ 0.01 compared with vehicle-treated control in a two-tailed Student's $t$ test.

To explore the mechanism of PROTAC(H-PGDS)-1-induced degradation of the H-PGDS protein, we first examined the effect of TFC-007 and pomalidomide on the H-PGDS protein. While addition of PROTAC(H-PGDS)-1 resulted in dramatic degradation of H-PGDS protein, the combination of TFC-007 and pomalidomide $(1 \mu \mathrm{M}$ each) did not effectively decrease the amount of H-PGDS protein (Figure 3a), indicating that linking the two ligands into a single molecule is critically important for the degradation of the H-PGDS protein. PROTAC(H-PGDS)-1 was designed to recruit CRBN for the degradation of H-PGDS protein. A competition assay using an excess amount of pomalidomide diminished the protein degradation activity of PROTAC(H-PGDS)-1 (Figure 3b), indicating that CRBN binding was required for the protein degradation.

To investigate the involvement of the UPS in the degradation of H-PGDS protein by PROTAC(H-PGDS)-1, we used the proteasome inhibitor MG132 and the ubiquitin-activating enzyme inhibitor MLN7243. The PROTAC(H-PGDS)-1-induced degradation of H-PGDS protein was suppressed by the inhibitors (Figure 3c), suggesting that the degradation of H-PGDS protein requires the UPS. 
(a)
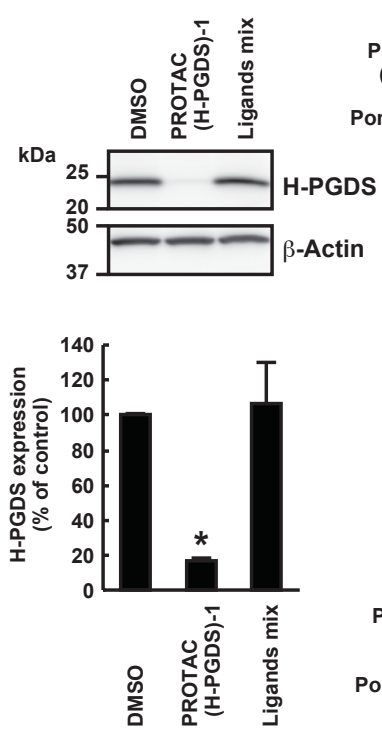

(b)

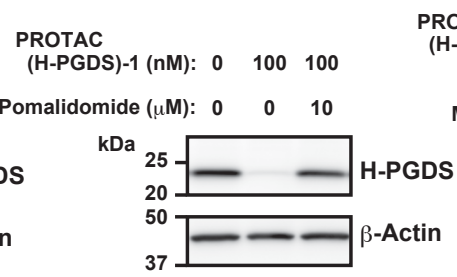

(c)
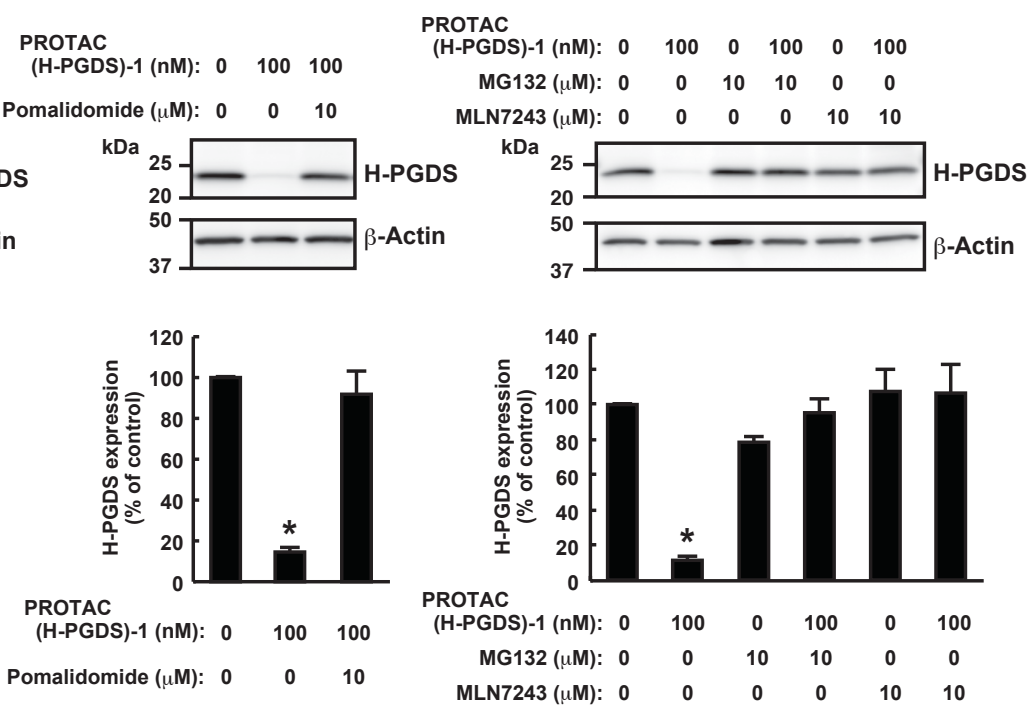

Figure 3. Involvement of the ubiqutin-proteasome system in the PROTAC(H-PGDS)-1-induced degradation of H-PGDS protein. (a) KU812 cells were incubated with $1 \mu \mathrm{M}$ PROTAC(H-PGDS)-1 or the ligand mixture (TFC-007 and pomalidomide, $1 \mu \mathrm{M}$ each) for $6 \mathrm{~h}$. (b) Competition assay using an excess amount of pomalidomide with PROTAC(H-PGDS)-1 in KU812 cells. Cells were incubated with $100 \mathrm{nM}$ PROTAC(H-PGDS)-1 and/or $10 \mu \mathrm{M}$ pomalidomide for $6 \mathrm{~h}$. (c) Effect of MG132 and MLN7243 on the protein knockdown activity of PROTAC(H-PGDS)-1 in KU812 cells. Cells were incubated with 100 nM PROTAC(H-PGDS)-1 in the presence or absence of $10 \mu \mathrm{M}$ MG132 or $10 \mu \mathrm{M}$ MLN7243 for $6 \mathrm{~h}$. The H-PGDS/ $\beta$-Actin ratio was normalized by the vehicle control as 100 . Data in the bar graphs are the mean $\pm \mathrm{SD}$ $(n=3) . * P<0.01$ compared to vehicle-treated control in a two-tailed Student's t test. 
As discussed above, PROTAC(H-PGDS)-1 is a potent H-PGDS protein degrader that is dependent on the UPS. In addition to the degradation, PROTAC(H-PGDS)-1 may also inhibit the enzymatic activity of H-PGDS; this is because it contains the TFC-007 moiety, which inhibits H-PGDS enzymatic activity. To investigate the importance of H-PGDS degradation for the suppression of $\mathrm{PGD}_{2}$ production, we developed a structurally related inactive degrader as a control compound. PROTAC(H-PGDS)-2 is composed of TFC-007 and $N$-methylated pomalidomide, which is unable to recruit CRBN. ${ }^{12}$ Using fluorescence polarization assays, we investigated the binding affinity to H-PGDS. TFC-007, PROTAC(H-PGDS)-1, and PROTAC(H-PGDS)-2 showed similar affinity toward H-PGDS with $\mathrm{IC}_{50}$ values of $0.32,0.32$, and $0.30 \mu \mathrm{M}$, respectively (Figure $4 \mathrm{a}$ ). We also investigated the inhibitory activity of the compounds against the H-PGDS enzyme in vitro. PROTAC(H-PGDS)-1 and PROTAC(H-PGDS)-2 had slightly lower $\mathrm{IC}_{50}$ values, $266 \mathrm{nM}$ for PROTAC(H-PGDS)-1 and $320 \mathrm{nM}$ for PROTAC(H-PGDS)-2, than TFC-007 (71 nM, Figure S4). Then, we examined the degradation activity of the compounds against H-PGDS protein. KU812 cells were treated with PROTAC(H-PGDS)-1, PROTAC(H-PGDS)-2, or TFC-007 for 24 h. PROTAC(H-PGDS)-1 reduced H-PGDS protein levels, while neither PROTAC(H-PGDS)-2 nor TFC-007 affected H-PGDS protein levels (Figure 4b). Taken together, these results indicated that PROTAC(H-PGDS)-1 was a potent degrader and inhibitor of H-PGDS, while PROTAC(H-PGDS)-2 and TFC-007 inhibited enzymatic activity but did not degrade H-PGDS. Finally, we investigated the effect of PROTAC(H-PGDS)-1, PROTAC(H-PGDS)-2, and TFC-007 on the production of $\mathrm{PGD}_{2}$ in KU812 cells. Treatment with the compounds for $24 \mathrm{~h}$ suppressed the production of $\mathrm{PGD}_{2}$, and 
PROTAC(H-PGDS)-1 reduced $\mathrm{PGD}_{2}$ production more effectively than the other compounds (Figure 4c).

(a)

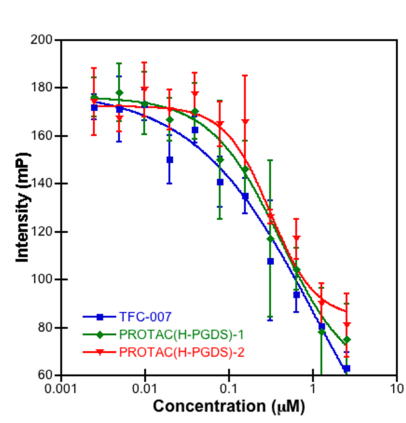

(b)

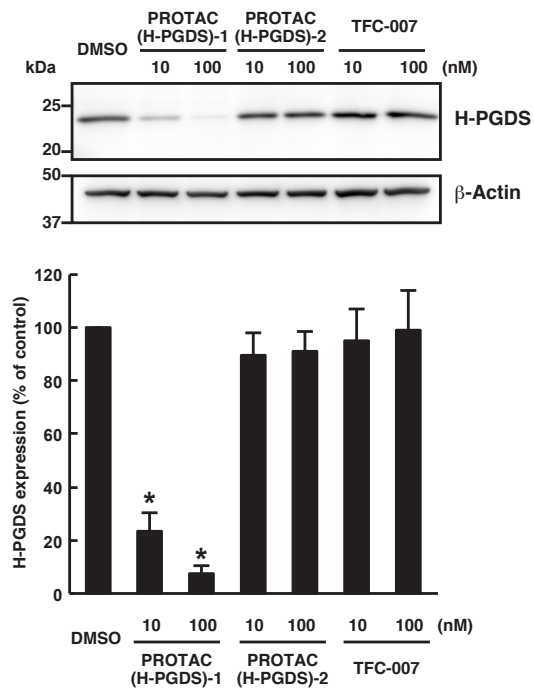

(c)

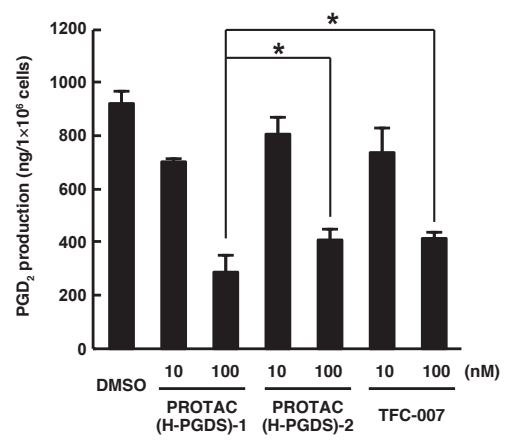

Figure 4. Comparison of the degradation and inhibition of H-PGDS. (a) Fluorescence polarization assays of the binding affinity between H-PGDS and TFC-007, PROTAC(H-PGDS)-1, and PROTAC(H-PGDS)-2. (b) KU812 cells were incubated with the indicated concentration of the compounds for $24 \mathrm{~h}$. The H-PGDS/ $\beta$-Actin ratio was normalized by the vehicle control as 100 . Data in the bar graph are the mean \pm SD $(n=3) . * P<0.01$ compared to vehicle-treated control in a two-tailed Student's t test. (c) KU812 cells were incubated with the indicated concentration of the compounds for $24 \mathrm{~h}$ and then incubated with $5 \mu \mathrm{M}$ A23187 in the presence of each compound for $30 \mathrm{~min}$. $\mathrm{PGD}_{2}$ levels in the medium were measured. Data in the bar graph are the mean $\pm \mathrm{SD}(n$ $=3) .{ }^{*} P<0.05$ in a two-tailed Student's t test. 
In conclusion, a potent degrader of the H-PGDS protein, PROTAC(H-PGDS)-1, was successfully developed by conjugating TFC-007 (H-PGDS ligand) to pomalidomide (E3 ligase, CRBN ligand). PROTAC(H-PGDS)-1 effectively induced the selective degradation of H-PGDS protein via the UPS and significantly suppressed $\mathrm{PGD}_{2}$ production. The degrader PROTAC(H-PGDS)-1 with a new mechanism of action is expected to be as effective, or more effective, than conventional inhibitors and should allow a reduction in the number of doses for the treatment of chronic inflammation. To clinically develop an H-PGDS degrader, we need to determine the on- and off-target effects, and to investigate the ADMET (absorption, distribution, metabolism, excretion, and toxicity) aspects of the H-PGDS degrader. Further structure-based improvement of the H-PGDS degraders is in progress in our laboratory.

\section{Methods}

\section{Synthesis and characterization of H-PGDS degraders}

We designed hybrid molecules, in which an H-PGDS inhibitor was linked to a ligand for CRBN or IAPs via a linker containing a polyethylene glycerol unit. The chemical synthesis and physicochemical data of the compounds are provided in the supporting information.

\section{Reagents}

Tissue culture plastics were purchased from Greiner Bio-One (Frickenhausen, Germany). Cycloheximide was purchased from Sigma-Aldrich (St. Louis, MO, USA). MG132 was purchased from Peptide Institute (Osaka, Japan). MLN7243 was purchased from Active Biochem (Maplewood, NJ, USA). Pomalidomide was purchased from 
Cayman Chemical (Ann Arbor, MI, USA). A23187 was purchased from Merck KGaA (Darmstadt, Germany).

\section{Cell culture}

Human chronic myelogenous leukemia KU812 cells were cultured in Roswell Park Memorial Institute-1640 (RPMI-1640) medium (Sigma-Aldrich, St. Louis, MO, USA) supplemented with 10\% fetal bovine serum (FBS) (Thermo Fisher Scientific, Waltham, MA, USA), and $100 \mu \mathrm{g} / \mathrm{mL}$ kanamycin (Sigma-Aldrich). Human megakaryoblastic leukemia MEG-01s cells were cultured in Dulbecco's Modified Eagle's medium (Sigma-Aldrich) supplemented with 10\% FBS and $100 \mu \mathrm{g} / \mathrm{mL}$ kanamycin. KU812 cells were obtained from the Japanese Collection of Research Bioresources (Osaka, Japan) Cell Bank (JCRB0104). ${ }^{21}$

\section{Western blot analysis}

Cells were lysed with SDS lysis buffer $(0.1 \mathrm{M}$ Tris- $\mathrm{HCl}$ at $\mathrm{pH} 8.0,10 \%$ glycerol, and $1 \%$ SDS). Protein concentration was measured by the BCA method (Thermo Fischer Scientific), and an equal amount of protein lysate was separated by SDS-PAGE, transferred to PVDF membranes (Millipore, County Cork, Ireland), and analyzed by western blot using the appropriate antibodies. The immunoreactive proteins were visualized using Clarity Western ECL substrate (Bio-Rad, Hercules, CA, USA), and their light emission was quantified with a LAS-3000 lumino-image analyzer (Fuji, Tokyo, Japan). The following antibodies were used: anti-H-PGDS rabbit polyclonal antibody (pAb); ${ }^{22}$ anti-mPGES-1 mouse monoclonal antibody (mAb); ${ }^{23}$ anti-CRBN rabbit pAb (\#7180) (Cell Signaling Technology, Danvers, MA, USA); anti- $\beta$-Actin 
mouse mAb (A2228) (Sigma-Aldrich); anti-Cyclin B1 mouse mAb (sc-245 HRP) (Santa Cruz, Dallas, TX, USA); and anti-cIAP1 goat pAb (AF8181) (R\&D Systems, Minneapolis, MN, USA).

\section{RNA isolation and quantitative PCR}

Total RNA was prepared from cells using a RNeasy kit (Qiagen, Hilden, Germany). First-strand cDNA was synthesized from $1 \mu \mathrm{g}$ of total RNA with an oligo-dT primer using the SuperScript First-Strand Synthesis System (Invitrogen, Carsbad, CA, USA). Quantitative real-time PCR was performed with an ABI Prism 7300 sequence detection system (Applied Biosystems, Foster City, CA, USA) using SYBR GreenER (Invitrogen) with gene-specific primers. Human 36B4 mRNA was used as an invariant control. The following PCR primers were used $\left(5^{\prime}\right.$ to $\left.3^{\prime}\right)$ : $36 B 4$, GGCCCGAGAAGACCTCCTT and CCAGTCTTGATCAGCTGCACA; H-PGDS, TGCCGTCGCTAACTGGATAA and GAGATGCCCCCGAGAAAAAC.

\section{Binding affinity to H-PGDS}

The fluorescence polarization (FP) assay was performed using a Prostaglandin D Synthase (hematopoietic-type) FP-Based Inhibitor Screening Assay Kit - Green (600007) (Cayman Chemical).

\section{Expression and Purification of recombinant H-PGDS}

Human H-PGDS were expressed and purified as described previously. ${ }^{6}$ In brief, E. coli BL21 (DE3) cells (Thermo Fisher Scientific) were transformed with the prepared plasmids. The cells were grown in LB medium (Nacalai Tesque, Kyoto, Japan) at $37^{\circ} \mathrm{C}$, 
induced with $600 \mu \mathrm{M}$ isopropyl $\beta$-D-1-thiogalactopyranoside (Nakalai Tesque), and then cultured further for $4 \mathrm{~h}$ at $37^{\circ} \mathrm{C}$. The cells were collected and disrupted by sonication in PBS containing a protease inhibitor cocktail (Roche, Basel, Switzerland). After removal of cell debris by centrifugation, the supernatant was filtered and applied onto a GSH-Sepharose 4B column (GE Healthcare UK Ltd., Buckinghamshire, England). After the resin had been washed with PBS, the protein adsorbed on the GSH-Sepharose 4B was eluted with $50 \mathrm{mM}$ Tris- $\mathrm{HCl}$ (pH 9.0) containing $10 \mathrm{mM} \mathrm{GSH}$. Protein concentration was determined by the BCA method (Thermo Fischer Scientific).

\section{Inhibitory activity against H-PGDS}

The activity of recombinant H-PGDS was measured by the spectrophotometric method with $1 \mathrm{mM}$ 1-chloro-2,4-dinitrobenzene (Sigma-Aldrich), $1 \mathrm{mM}$ glutathione (Sigma-Aldrich), and $2.8 \mu \mathrm{g} / \mathrm{mL}$ recombinant human H-PGDS in $100 \mathrm{mM}$ Tris-HCl ( $\mathrm{pH}$ 8.0) in the presence $0.1 \mathrm{mg} / \mathrm{ml}$ IgG (rabbit $\gamma$-goblin, Sigma-Aldrich). The reactions were monitored in a 96 well microplate, and the enzymatic product was followed at $340 \mathrm{~nm}$ over $3 \mathrm{~min}$ at $25{ }^{\circ} \mathrm{C}$ using a microplate spectrophotometer (Multiskan FC, Thermo Fisher Scientific). The $\mathrm{IC}_{50}$ values were calculated using GraphPad Prism software (version 5 for Windows).

\section{Measurement of PG levels}

$\mathrm{PGD}_{2}$ levels in the medium were measured by using a $\mathrm{PGD}_{2}$ MOX ELISA Kit (512011) (Cayman Chemical) according to the manufacturer's instructions. The absorbance at $405 \mathrm{~nm}$ was measured using an EnVision Multilabel Plate Reader (PerkinElmer, Waltham, MA, USA). 


\section{Statistical analysis}

Two-tailed Student's t tests were used to determine the significance of differences between experimental groups.

\section{Acknowledgements}

Victoria Muir, PhD, from Edanz Group (https://en-author-services.edanzgroup.com/ac) edited a draft of this manuscript.

\section{Funding Sources}

This study was supported in part by grants from AMED under Grant Number 20mk0101120j0003 to Y.D., 20ak0101073j0604 to M.N., and 20ak0101073j0904 to Y.D.; JSPS/MEXT (KAKENHI, Grants JP18K07311 to N.S., JP17K08385 to Y.D., and JP18H05502 to M.N. and Y.D.); TERUMO FOUNDATION for life sciences and ARTS (to Y.D.); Takeda Science Foundation (to N.S. and Y.D.); the Naito Foundation (to Y.D.); the Sumitomo Foundation (to Y.D.); and the Novartis Foundation (Japan) for the Promotion of Science (to Y.D.).

\section{References}

1. Lewis, R. A., Soter, N. A., Diamond, P. T., Austen, K. F., Oates, J. A. and Roberts, L. J., 2nd (1982) Prostaglandin D2 generation after activation of rat and human mast cells with anti-IgE. J. Immunol. 129, 1627-1631.

2. Matsuoka, T., Hirata, M., Tanaka, H., Takahashi, Y., Murata, T., Kabashima, K., Sugimoto, Y., Kobayashi, T., Ushikubi, F., Aze, Y., Eguchi, N., Urade, Y., Yoshida, 
N., Kimura, K., Mizoguchi, A., Honda, Y., Nagai, H. and Narumiya, S. (2000) Prostaglandin D2 as a mediator of allergic asthma. Science 287, 2013-2017.

3. Urade, Y. and Hayaishi, O. (2000) Biochemical, structural, genetic, physiological, and pathophysiological features of lipocalin-type prostaglandin D synthase. Biochim. Biophys. Acta 1482, 259-271.

4. Takeshita, E., Komaki, H., Shimizu-Motohashi, Y., Ishiyama, A., Sasaki, and M., Takeda, S. (2018) A phase I study of TAS-205 in patients with Duchenne muscular dystrophy. Ann. Clin. Transl. Neurol. 5, 1338-1349.

5. Rittchen, S., and Heinemann, A. (2019) Therapeutic Potential of Hematopoietic Prostaglandin D2 Synthase in Allergic Inflammation Cells 8, 619.

6. Aritake, K., Kado, Y., Inoue, T., Miyano, M., and Urade, Y. (2006) Structural and functional characterization of HQL-79, an orally selective inhibitor of human hematopoietic prostaglandin D synthase. J. Biol. Chem. 281, 15277-15286.

7. Kajiwara, D., Aoyagi, H., Shigeno, K., Togawa, M., Tanaka, K., Inagaki, N., and Miyoshi, K. (2011) Role of hematopoietic prostaglandin D synthase in biphasic nasal obstruction in guinea pig model of experimental allergic rhinitis. Eur. J. Pharmacol. 667, 389-395.

8. Nabe, T., Kuriyama, Y., Mizutani, N., Shibayama, S., Hiromoto, A., Fujii, M., Tanaka, K., and Kohno, S. (2011) Inhibition of hematopoietic prostaglandin D synthase improves allergic nasal blockage in guinea pigs. Prostaglandins Other Lipid Mediat. 95, 27-34.

9. Hughes, S. J., and Ciulli, A. (2017) Molecular recognition of ternary complexes: a new dimension in the structure-guided design of chemical degraders. Essays Biochem 61, 505-516. 
10. Lai, A. C., and Crews, C. M. (2017) Induced protein degradation: an emerging drug discovery paradigm. Nat. Rev. Drug Discov. 16, 101-14.

11. Ohoka, N., Shibata, N., Hattori, T., and Naito, M. (2016) Protein Knockdown Technology: Application of Ubiquitin Ligase to Cancer Therapy. Curr. Cancer Drug Targets 16, 136-146.

12. Lu, J., Qian, Y., Altieri, M., Dong, H., Wang, J., Raina, K., Hines, J., Winkler, J. D., Crew, A. P., Coleman, K., and Crews, C. M. (2015) Hijacking the E3 Ubiquitin Ligase Cereblon to Efficiently Target BRD4. Chem. Biol. 22, 755-763. doi: 10.1016/j.chembiol.2015.05.009.

13. Shibata, N., Shimokawa, K., Nagai, K., Ohoka, N., Hattori, T., Miyamoto, N., Ujikawa, O., Sameshima, T., Nara, H., Cho, N., and Naito, M. (2018) Pharmacological difference between degrader and inhibitor against oncogenic BCR-ABL kinase. Sci Rep., 813549.

14. You, I., Erickson, E. C., Donovan, K. A., Eleuteri, N. A., Fischer, E. S., Gray, N.S., and Toker, A. (2020) Discovery of an AKT Degrader with Prolonged Inhibition of Downstream Signaling. Cell Chem. Biol., 27, 66-73.

15. Burslem., G. M., Crews, C. M. (2020) Proteolysis-Targeting Chimeras as Therapeutics and Tools for Biological Discovery. Cell. 181,102-114.

16. Pei, H., Peng, Y., Zhao, Q., and Chen, Y. (2019) Small molecule PROTACs: an emerging technology for targeted therapy in drug discovery. RSC Adv. 9, $16967-16976$

17. Matsushita, N., Aritake, K., Takada, A., Hizue, M., Hayashi, K., Mitsui, K., Hayashi, M., Hirotsu, I., Kimura, Y., Tani, T., and Nakajima, H. (1998) Pharmacological Studies on the Novel Antiallergic Drug HQL-79: II. 
Elucidation of Mechanisms for Antiallergic and Antiasthmatic Effects. Jpn. J. Pharmacol. 78, 11-22.

18. Takaya, D., Inaka, K., Omura, A., Takenuki, K., Kawanishi, M., Yabuki, Y., Nakagawa, Y., Tsuganezawa, K., Ogawa, N., Watanabe, C., Honma, T., Aritake, K., Urade, Y., Shirouzu, M., and Tanaka, A. (2018) Characterization of crystal water molecules in a high-affinity inhibitor and hematopoietic prostaglandin D synthase complex by interaction energy studies. Bioorg. Med. Chem. 26, $4726-4734$.

19. Inoue, T., Okano, Y., Kado, Y., Aritake, K., Irikura, D., Uodome, N., Okazaki, N., Kinugasa, S., Shishitani, H., Matsumura, H., Kai, Y. and Urade, Y. (2004) First determination of the inhibitor complex structure of human hematopoietic prostaglandin D synthase. J. Biochem. 135, 279-283.

20. Hara, S., Kamei, D., Sasaki, Y., Tanemoto, A., Nakatani, Y., and Murakami, M. (2010) Prostaglandin E synthases: understanding their pathophysiological roles through mouse genetic models. Biochimie. 92, 651-659

21. Kishi, K. (1985) A new leukemia cell line with Philadelphia chromosome characterized as basophil precursors. Leuk. Res. 9, 381-390.

22. Mohri, I., Eguchi, N., Suzuki, K., Urade, Y., and Taniike, M. (2003) Hematopoietic prostaglandin D synthase is expressed in microglia in the developing postnatal mouse brain. Glia. 42, 263-274.

23.Lazarus, M., Eguchi, N., Matsumoto, S., Nagata, N., Yano, T., Killian, G. J., and Urade, Y. (2004) Species-specific expression of microsomal prostaglandin E synthase-1 and cyclooxygenases in male monkey reproductive organs. Prostaglandins Leukot. Essent. Fatty Acids. 71, 233-240. 